\title{
SWIFFT: A Modest Proposal for FFT Hashing ${ }^{\star}$
}

\author{
Vadim Lyubashevsky ${ }^{1}$, Daniele Micciancio ${ }^{1}$, Chris Peikert $^{2, \star \star}$, \\ and Alon Rosen ${ }^{3}$ \\ ${ }^{1}$ University of California at San Diego \\ ${ }^{2}$ SRI International \\ ${ }^{3}$ IDC Herzliya
}

\begin{abstract}
We propose SWIFFT, a collection of compression functions that are highly parallelizable and admit very efficient implementations on modern microprocessors. The main technique underlying our functions is a novel use of the Fast Fourier Transform (FFT) to achieve "diffusion," together with a linear combination to achieve compression and "confusion." We provide a detailed security analysis of concrete instantiations, and give a high-performance software implementation that exploits the inherent parallelism of the FFT algorithm. The throughput of our implementation is competitive with that of SHA-256, with additional parallelism yet to be exploited.

Our functions are set apart from prior proposals (having comparable efficiency) by a supporting asymptotic security proof: it can be formally proved that finding a collision in a randomly-chosen function from the family (with noticeable probability) is at least as hard as finding short vectors in cyclic/ideal lattices in the worst case.
\end{abstract}

\section{Introduction}

In cryptography, there has traditionally been a tension between efficiency and rigorous security guarantees. The vast majority of proposed cryptographic hash functions have been designed to be highly efficient, but their resilience to attacks is based only on intuitive arguments and validated by intensive cryptanalytic efforts. Recently, new cryptanalytic techniques [29304] have started casting serious doubts both on the security of these specific functions and on the effectiveness of the underlying design paradigm.

On the other side of the spectrum, there are hash functions having rigorous asymptotic proofs of security (i.e., security reductions), assuming that various computational problems (such as the discrete logarithm problem or factoring large integers) are hard to solve on the average. Unfortunately, all such proposed hash functions have had computation cost comparable to typical public key cryptographic operations, making them unattractive from a practical point of view.

* Mod·est, adj.: Marked by simplicity.

${ }^{\star \star}$ Supported by the National Science Foundation under Grants CNS-0716786 and CNS-0749931. Any opinions, findings, and conclusions or recommedations expressed in this material are those of the author(s) and do not necessarily reflect the views of the National Science Foundation. 


\subsection{Our Proposal: SWIFFT}

We propose the SWIFFT collection of compression functions, and give a highperformance software implementation. SWIFFT is very appealing and intuitive from a traditional design perspective, and, at the same time, achieves the robustness and reliability benefits of provable asymptotic security under a mild computational assumption. The functions correspond to a simple algebraic expression over a certain polynomial ring, as described in detail in Section 2.1 Here we describe a high-level algorithm for the fast evaluation of a SWIFFT compression function.

The algorithm takes as input a binary string of length $m n$ (for suitable parameters $m, n)$, which is viewed as an $n \times m$ binary matrix $\left(x_{i, j}\right) \in\{0,1\}^{n \times m}$. It then performs the following two steps, where all operations are performed in $\mathbb{Z}_{p}$ for an appropriate modulus $p$ :

1. The input matrix $\left(x_{i, j}\right)$ is first processed by multiplying the $i$ th row by $\omega^{i-1}$ for $i=1, \ldots, n$ (where $\omega \in \mathbb{Z}_{p}$ is an appropriate fixed element).

Then the Fast Fourier Transform (FFT) is computed (over $\mathbb{Z}_{p}$ ) on each column $j=1, \ldots, m$ :

$$
\left(y_{1, j}, \ldots, y_{n, j}\right)=\operatorname{FFT}\left(\omega^{0} \cdot x_{1, j}, \ldots, \omega^{n-1} \cdot x_{n, j}\right) .
$$

We remark that this operation is easy to invert, and is performed to achieve "diffusion," i.e., to mix the input bits of every column.

2. A linear combination is then computed across each row $i=1, \ldots, n$ :

$$
z_{i}=a_{i, 1} \cdot y_{i, 1}+\cdots+a_{i, m} \cdot y_{i, m}=\sum_{j=1}^{m} a_{i, j} \cdot y_{i, j},
$$

where the coefficients $a_{i, j} \in \mathbb{Z}_{p}$ are fixed as part of the function description. This operation compresses the input, achieving "confusion."

The output is the vector $\left(z_{1}, \ldots, z_{n}\right) \in \mathbb{Z}_{p}^{n}$.

Consider an attempt to invert the function, i.e., to find some input $\left(x_{i, j}\right)$ that evaluates to a given output $\left(z_{1}, \ldots, z_{n}\right)$. Viewed independently, each linear equation $z_{i}=\sum_{j=1}^{m} a_{i, j} \cdot y_{i, j}$ on the rows admits a large number of easily-computed solutions. However, there are strong dependencies among the equations. In particular, every column $\left(y_{1, j}, \ldots, y_{n, j}\right)$ is constrained to be the result of applying Step 1 to an $n$-dimensional binary vector $\left(x_{1, j}, \ldots, x_{n, j}\right) \in\{0,1\}^{n}$.

Perhaps surprisingly, these constraints turn out to be sufficient to guarantee asymptotically that the SWIFFT functions are provably one-way and collisionresistant. More precisely, the family admits a very strong security reduction: finding collisions on the average (when the coefficients $a_{i, j}$ are chosen at random in $\mathbb{Z}_{p}$ ) with any noticeable probability is at least as hard as solving an underlying mathematical problem on certain kinds of point lattices in the worst case. This claim follows from the fact that the SWIFFT functions are a special case of the cyclic/ideal lattice-based functions of [18/2116. 
SWIFFT's simple design has a number of other advantages. First, it also enables unconditional proofs of a variety of statistical properties that are desirable in many applications of hash functions, both in cryptography and in other domains. Second, its underlying mathematical structure is closely related to wellstudied cryptographic problems, which permits easy understanding and analysis of concrete instantiations. Third, it is extremely parallelizable, and admits software implementations with throughput comparable to (or even exceeding) the SHA-2 family on modern microprocessors.

While SWIFFT satisfies many desirable cryptographic and statistical properties, we caution that it was not designed to be an "all-purpose" cryptographic hash function. For example, it is not (by itself) a pseudorandom function, and would not be a suitable instantiation of a random oracle. (See Section 4 for more details.) In addition, while the concrete parameters were chosen so as to resist all known feasible attacks, SWIFFT does not achieve full "birthday bound" security of $2^{n / 2}$ for collision attacks with an $n$-bit output, nor $2^{n}$ security for inversion attacks. (See Section 5 for more details.)

\subsection{Related Work}

Using the Fast Fourier Transform (FFT) as a building block in hash functions is not new. For example, Schnorr et al proposed a variety of FFT-based hash functions 24 2526, which unfortunately were subsequently cryptanalyzed and shown to be insecure 922 27. Our compression functions are set apart from previous work by the way that the FFT is used, and the resulting proof of security. Namely, while in previous work 24 25 26] the FFT was applied to unrestricted input vectors $\left(x_{1}, \ldots, x_{n}\right) \in \mathbb{Z}_{p}^{n}$, here we require the input values $x_{i}$ to be bits. This introduces non-linear constraints on the output values of the FFT operation, a fact that plays a fundamental role both in our theoretical proof of security, as well as on the analysis of our concrete functions. Our novel use of FFT may be of independent interest, and might find other applications in cryptographic design.

The subset-sum and knapsack problems have long ago been suggested as foundations for compression functions, e.g., by Damgård [10]. Unfortunately, these functions are only efficient in small dimensions, at which point lattice-based attacks [14 and other forms of cryptanalysis [7] become possible.

An important ingredient in the conceptual design of our functions (and associated proof of security) is the use of lattices with special structure as an underlying mathematical problem. Special classes of lattices (with closely related, but somewhat different structure than ours) also have been used before in practical constructions (most notably, the NTRU encryption scheme [13] and LASH hash function [3]), but without any security proofs.

Most closely related to our work is the theoretical study initiated by Ajtai [1] of subset sum-like cryptographic functions that are provably secure under worst-case assumptions for lattice problems. Ajtai's work and subsequent improvements [1161719 do not lead to very efficient implementations, mostly because of the huge size of the function description and slow evaluation time 
(which grow quadratically in the security parameter). A first step toward bridging the gap between theoretical constructions and practical functions was taken by Micciancio 18, who proposed using lattices with special structure (namely, cyclic lattices) and showed how they lead to cryptographic functions that have provable worst-case hardness and also admit fast implementations using FFT. The main limitation of the functions proposed in [18] was the notion of security achieved: they are provably one-way (under a worst-case assumption on cyclic lattices), but not collision resistant. Peikert and Rosen 21] and Lyubashevsky and Micciancio [16] then modified and generalized the function originally proposed in [18] to achieve collision resistance.

From a theoretical point of view, the SWIFFT functions proposed in this paper are equivalent to and inherit all provable security features from the cyclic/ ideal hash functions of [21]6. But differently from [18/21]6], the emphasis in this paper is on practical implementation issues, and the construction of concrete instances and variants of those functions that enjoy very efficient implementation from a practical point of view. For a deeper understanding of the theoretical ideas underlying the proofs of security of our compression functions, we refer the reader to 182116 .

\section{SWIFFT Compression Functions}

In this section, we describe an algebraic expression that is the underlying foundation of the SWIFFT functions, and how it is related to the FFT-based algorithm described in Section 1.1. We then propose a set of concrete parameters on which our implementation and security analysis are based.

\subsection{Algebraic Description}

The SWIFFT functions correspond to a simple algebraic expression over a certain polynomial ring. A family of SWIFFT functions is described by three main parameters: let $n$ be a power of 2 , let $m>0$ be a small integer, and let $p>0$ be a modulus (not necessarily prime, though we will soon see that certain prime $p$ will be convenient). Define $R$ to be the ring $R=\mathbb{Z}_{p}[\alpha] /\left(\alpha^{n}+1\right)$, i.e., the ring of polynomials (in $\alpha$ ) having integer coefficients, modulo $p$ and $\alpha^{n}+1$. Any element of $R$ may therefore be written as a polynomial of degree $<n$ having coefficients in $\mathbb{Z}_{p}=\{0, \ldots, p-1\}$.

A particular function in the family is specified by $m$ fixed elements $\mathbf{a}_{1}, z \ldots$, $\mathbf{a}_{m} \in R$ of the ring $R$, called "multipliers." The function corresponds to the following expression over the ring $R$ :

$$
\sum_{i=1}^{m}\left(\mathbf{a}_{i} \cdot \mathbf{x}_{i}\right) \in R
$$

where $\mathbf{x}_{1}, \ldots, \mathbf{x}_{m} \in R$ are polynomials having binary coefficients, and corresponding to the binary input of length $m n$. 
To compute the above expression, the main bottleneck is in computing the polynomial products $\mathbf{a}_{i} \cdot \mathbf{x}_{i}$ over $R$. It is well-known that the Fast Fourier Transform (FFT) provides an $O(n \log n)$-time algorithm that can be used for multiplying polynomials of degree $<n$. The multiplication algorithm starts by using the FFT to compute (all at once) the Fourier coefficients of each polynomial, i.e., the values on all the $2 n$th roots of unity over the complex field $\mathbb{C}$. It then multiplies the respective Fourier coefficients of the two polynomials, and finally interpolates back to a degree $<2 n$ polynomial via an inverse FFT.

Because we are working modulo $p$ and $\alpha^{n}+1$, there is an even more convenient and efficient method for computing the polynomial products in the ring $R$. Suppose that $p$ is prime and $p-1$ is a multiple of $2 n$. Then $\mathbb{Z}_{p}$ is a field, and it contains a multiplicative subgroup of order $2 n$ whose elements are all the $2 n$th roots of unity in $\mathbb{Z}_{p}$ (i.e., the roots of the polynomial $\alpha^{2 n}-1 \bmod p$ ). Let $\omega \in \mathbb{Z}_{p}$ be some generator of this subgroup, i.e., an element of order $2 n$. The $n$ odd powers $\omega^{1}, \omega^{3}, \ldots, \omega^{2 n-1}$ are exactly the primitive $2 n$th roots of unity, i.e., the roots of $\alpha^{n}+1$.

In order to compute a polynomial product $\mathbf{a}_{i} \cdot \mathbf{x}_{i}$ modulo $p$ and $\alpha^{n}+1$, it suffices to compute only the $n$ primitive Fourier coefficients of $\mathbf{a}_{i}$ and $\mathbf{x}_{i}$, i.e., the values $\mathbf{a}_{i}\left(\omega^{1}\right), \mathbf{a}_{i}\left(\omega^{3}\right), \ldots, \mathbf{a}_{i}\left(\omega^{2 n-1}\right)$, and likewise for $\mathbf{x}_{i}$. The primitive coefficients can be computed all at once by preprocessing the input and then applying an $n$-dimensional FFT (which uses half the space), as described in the algorithm from Section 1.1. Furthermore, because the field $\mathbb{Z}_{p}$ has roots of unity, the FFT can be performed over $\mathbb{Z}_{p}$ using the $n$th primitive root of unity $\omega^{2}$, instead of over $\mathbb{C}$. 1

In addition to using an FFT, other significant optimizations are possible when computing Expression (10). First, because the multipliers $\mathbf{a}_{i}$ are fixed in advance and determined uniquely by their primitive Fourier coefficients, we can simply store and work with their Fourier representation. Additionally, because the FFT is linear and a bijection, there is no need to even apply an inverse FFT. In other words, the value of Expression (11) is correctly and uniquely determined by summing the Fourier representations of $\mathbf{a}_{i} \cdot \mathbf{x}_{i}$. Combining all these observations, we are left with the high-level algorithm as described in Section 1.1. which we implement (using additional optimizations) in Section 3 .

\subsection{Concrete Parameters}

In this paper we primarily study one family of SWIFFT compression functions, obtained by choosing concrete values for the parameters $n, m$, and $p$ as follows:

$$
n=64, \quad m=16, \quad p=257 .
$$

For these parameters, any fixed compression function in the family takes a binary input of length $m n=1024$ bits (128 bytes), to an output in the range $\mathbb{Z}_{p}^{n}$, which

\footnotetext{
${ }^{1}$ Performing an FFT over $\mathbb{Z}_{p}$ rather than $\mathbb{C}$ is often called a number theoretic transform (NTT) in the literature; however, we will retain the FFT terminology due to broad familiarity.
} 
has size $p^{n}=257^{64} \approx 2^{512}$. An output in $\mathbb{Z}_{p}^{n}$ can easily be represented using 528 bits ( 66 bytes). Other unambiguous representations (using $>512$ bits) are also possible; the representation does not affect security.

We now briefly explain our choice of parameters. The first consideration is the security of the compression function. As we will explain in the security analysis of Section 5. the function corresponds to a subset-sum from $m n$ bits to roughly $n \lg p$ bits. We first set the constraints $m n=1024$ and $n \lg p \approx 512$, because solving such subset-sum problems appears to be intractable. In order for our proofs of security to go through, we also need the polynomial $\alpha^{n}+1$ to be irreducible over $\mathbb{Z}[\alpha]$, which is true if and only if $n$ is a power of 2 . (If a reducible polynomial is used, actual attacks can become possible, as we show in Section 5.3 for similar functions in the literature.)

Next, we optimize the running time and space of the function by choosing $n$ to be relatively large, and $p$ and $m$ to be small, subject to the above constraints. As discussed above, the Fast Fourier Transform is most efficiently and conveniently computed when $p$ is prime and $p-1$ is a multiple of $2 n$.

Finally, to fix one concrete function from the family, the multipliers $\mathbf{a}_{i}$ should be chosen uniformly and independently at random from the ring $R$; this is equivalent to choosing their primitive Fourier coefficients uniformly and independently at random from $\mathbb{Z}_{p}$. We note that the multipliers (or their Fourier coefficients) should be chosen using "trusted randomness," otherwise it may be possible to embed a "backdoor" in the resulting function. For example, one might derive the multipliers using some deterministic transformation on the digits of $\pi$.

\section{Implementation}

Our implementation uses two main techniques for achieving high performance, both relating to the structure of the Fast Fourier Transform (FFT) algorithm. The first observation is that the input to the FFT is a binary vector, which limits the number of possible input values (when restricting our view to a small portion of the input). This allows us to precompute and store the results of several initial iterations of the FFT in a lookup table. The second observation is that the FFT algorithm consists of operations repeated in parallel over many pieces of data, for which modern microprocessors have special-purpose instruction sets.

Recall the parameters $n=64, m=16$, and modulus $p=257$. Let $\omega$ be a 128 th root of unity in $\mathbb{Z}_{p}=\mathbb{Z}_{257}$, i.e., an element of order $128=2 n$. (We will see later that it is convenient to choose $\omega=42$, but most of the discussion is independent from the choice of $\omega$.)

The compression function takes an $m n=1024$-bit input, viewed as $m=16$ binary vectors $\mathbf{x}_{0}, \ldots, \mathbf{x}_{15} \in\{0,1\}^{64}$. (For convenience, entries of a vector or sequence are numbered starting from 0 throughout this section.) The function first processes each vector $\mathbf{x}_{j}$, multiplying its $i$ th entry by $\omega^{i}$ (for $i=0, \ldots, 63$ ), and then computing the Fourier transform of the resulting vector using $\omega^{2}$ as a 64 th root of unity. More precisely, each input vector $\mathbf{x}_{j} \in\{0,1\}^{64}$ is mapped to 
$\mathbf{y}_{j}=F\left(\mathbf{x}_{j}\right)$, where $F:\{0,1\}^{64} \rightarrow \mathbb{Z}_{257}^{64}$ is the function

$$
F(\mathbf{x})_{i}=\sum_{k=0}^{63}\left(x_{k} \cdot \omega^{k}\right) \cdot\left(\omega^{2}\right)^{i \cdot k}=\sum_{k=0}^{63} x_{k} \cdot \omega^{(2 i+1) k} .
$$

The final output $\mathbf{z}$ of the compression function is then obtained by computing 64 distinct linear combinations (modulo 257) across the $i$ th entries of the $16 \mathbf{y}_{j}$ vectors:

$$
z_{i}=\sum_{j=0}^{15} a_{i, j} \cdot y_{i, j} \quad(\bmod 257),
$$

where the $a_{i, j} \in \mathbb{Z}_{257}$ are the primitive Fourier coefficients of the fixed multipliers.

Computing $F$. The most expensive part of the computation is clearly the computation of the transformation $F$ on the 16 input vectors $\mathbf{x}_{j}$, so we first focus on the efficient computation of $F$. Let $\mathbf{y}=F(\mathbf{x}) \in \mathbb{Z}_{257}^{64}$ for some $\mathbf{x} \in\{0,1\}^{64}$. Expressing the indices $i, k$ from Equation (2) in octal as $i=i_{0}+8 i_{1}$ and $k=k_{0}+8 k_{1}$ (where $\left.j_{0}, j_{1}, k_{0}, k_{1} \in\{0, \ldots, 7\}\right)$, and using $\omega^{128}=1(\bmod 257)$, the $i$ th component of $\mathbf{y}=F(\mathbf{x})$ is seen to equal

$$
\begin{aligned}
y_{i_{0}+8 i_{1}} & =\sum_{k_{0}=0}^{7}\left(\omega^{16}\right)^{i_{1} \cdot k_{0}}\left(\omega^{\left(2 i_{0}+1\right) k_{0}} \cdot \sum_{k_{1}=0}^{7} \omega^{8 k_{1}\left(2 i_{0}+1\right)} \cdot x_{k_{0}+8 k_{1}}\right) \\
& =\sum_{k_{0}=0}^{7}\left(\omega^{16}\right)^{i_{1} \cdot k_{0}}\left(m_{k_{0}, i_{0}} \cdot t_{k_{0}, i_{0}}\right),
\end{aligned}
$$

where $m_{k_{0}, i_{0}}=\omega^{\left(2 i_{0}+1\right) k_{0}}$ and $t_{k_{0}, i_{0}}=\sum_{k_{1}=0}^{7} \omega^{8 k_{1}\left(2 i_{0}+1\right)} x_{k_{0}+8 k_{1}}$. Our first observation is that each 8 -dimensional vector $\mathbf{t}_{k_{0}}=\left(t_{k_{0}, 0}, t_{k_{0}, 1}, \ldots, t_{k_{0}, 7}\right)$ can take only 256 possible values, depending on the corresponding input bits $x_{k_{0}}, x_{k_{0}+8}$, $\ldots, x_{k_{0}+8.7}$. Our implementation parses each 64 -bit block of the input as a sequence of 8 bytes $X_{0}, \ldots, X_{7}$, where $X_{k_{0}}=\left(x_{k_{0}}, x_{k_{0}+8}, \ldots, x_{k_{0}+8 \cdot 7}\right) \in\{0,1\}^{8}$, so that each vector $\mathbf{t}_{k_{0}}$ can be found with just a single table look-up operation $\mathbf{t}_{k_{0}}=T\left(X_{k_{0}}\right)$, using a table $T$ with 256 entries. The multipliers $\mathbf{m}_{k_{0}}=$ $\left(m_{k_{0}, 0}, \ldots, m_{k_{0}, 7}\right)$ can also be precomputed.

The value $\mathbf{y}=F(\mathbf{x})$ can be broken down as 8 (8-dimensional) vectors

$$
\mathbf{y}_{i_{1}}=\left(y_{8 i_{1}}, y_{8 i_{1}+1}, \ldots, y_{8 i_{1}+7}\right) \in \mathbb{Z}_{257}^{8} .
$$

Our second observation is that, for any $i_{0}=0, \ldots, 7$, the $i_{0}$ th component of $\mathbf{y}_{i_{1}}$ depends only on the $i_{0}$ th components of $\mathbf{m}_{k_{0}}$ and $\mathbf{t}_{k_{0}}$. Moreover, the operations performed for every coordinate are exactly the same. This permits parallelizing the computation of the output vectors $\mathbf{y}_{0}, \ldots, \mathbf{y}_{7}$ using SIMD (single-instruction multiple-data) instructions commonly found on modern microprocessors. For example, Intel's microprocessors (starting from the Pentium 4) include a set of so-called SSE2 instructions that allow operations on a set of special registers each holding an 8-dimensional vector with 16-bit (signed) integer components. 
We only use the most common SIMD instructions (e.g., component-wise addition and multiplication of vectors), which are also found on most other modern microprocessors, e.g., as part of the AltiVec SIMD instruction set of the Motorola G4 and IBM G5 and POWER6. In the rest of this section, operations on 8-dimensional vectors like $\mathbf{m}_{k_{0}}$ and $\mathbf{t}_{k_{0}}$ are interpreted as scalar operations applied component-wise to the vectors, possibly in parallel using a single SIMD instruction.

Going back to the computation of $F(\mathbf{x})$, the output vectors $\mathbf{y}_{i_{1}}$ can be expressed as

$$
\mathbf{y}_{i_{1}}=\sum_{k_{0}=0}^{7}\left(\omega^{16}\right)^{i_{1} \cdot k_{0}}\left(\mathbf{m}_{k_{0}} \cdot \mathbf{t}_{k_{0}}\right)
$$

Our third observation is that the latter computation is just a sequence of 8 component-wise multiplications $\mathbf{m}_{k_{0}} \cdot \mathbf{t}_{k_{0}}$, followed by a single 8-dimensional Fourier transform using $\omega^{16}$ as an 8 th root of unity in $\mathbb{Z}_{257}$. The latter can be efficiently implemented using a standard FFT network consisting of just 12 additions, 12 subtractions and 5 multiplications.

Optimizations relating to $\mathbb{Z}_{257}$. One last source of optimization comes from two more observations that are specific to the use of 257 as a modulus, and the choice of $\omega=42$ as a 128th root of unity. One observation is that the root used in the 8dimensional FFT computation equals $\omega^{16}=2^{2}(\bmod 257)$. So, multiplication by $\left(\omega^{16}\right),\left(\omega^{16}\right)^{2}$ and $\left(\omega^{16}\right)^{3}$, as required by the FFT, can be simply implemented as left bit-shift operations (by 2, 4, and 6 positions, respectively). Moreover, analysis of the FFT network shows that modular reduction can be avoided (without the risk of overflow using 16-bit arithmetic) for most of the intermediate values. Specifically, in our implementation, modular reduction is performed for only 3 of the intermediate values. The last observation is that, even when necessary to avoid overflow, reduction modulo 257 can be implemented rather cheaply and using common SIMD instructions, e.g., a 16-bit (signed) integer can be reduced to the range $\{-127, \ldots, 383\}$ using $x \equiv(x \wedge 255)-(x \gg 8) \bmod 257$, where $\wedge$ is the bit-wise "and" operation, and $\gg 8$ is a right-shift by 8 bits.

Summary and performance. In summary, function $F$ can be computed with just a handful of table look-ups and simple SIMD instructions on 8 dimensional vectors. The implementation of the remaining part of the computation of the compression function (i.e., the scalar products between $y_{i, j}$ and $a_{i, j}$ ) is straightforward, keeping in mind that this part of the computation can also be parallelized using SIMD instructions, and that reduction modulo 257 is rarely necessary during the intermediate steps of the computation due to the use of 16-bit (or larger) registers.

We implemented and tested our function on a $3.2 \mathrm{GHz}$ Intel Pentium 4. The implementation was written in $\mathrm{C}$ (using the Intel intrinsics to instruct the compiler to use SSE2 instructions), and compiled using gcc version 4.1.2 (compiler flags -O3) on a PC running under Linux kernel 2.6.18. Our tests show that our basic compression function can be evaluated in $1.5 \mu \mathrm{s}$ on the above system, 
yielding a throughput close to $40 \mathrm{MB} / \mathrm{s}$ in a standard chaining mode of operation. For comparison, we tested SHA256 on the same system using the highly optimized implementation in openssl version 0.9 .8 (using the openssl speed benchmark), yielding a throughput of $47 \mathrm{MB} / \mathrm{s}$ when run on $8 \mathrm{~KB}$ blocks.

Further optimizations. We remark that our implementation does not yet take advantage of all the potential for parallelism. In particular, we only exploited SIMD-level parallelism in individual evaluations of the transformation function $F$. Each evaluation of the compression function involves 16 applications of $F$, and subsequent multiplication of the result by the coefficients $a_{i, j}$. These 16 computations are completely independent, and can be easily executed in parallel on a multicore microprocessor. Our profiling data shows that the FFT computations and multiplication by $a_{i, j}$ currently account for about $90 \%$ of the running time. So, as multicore processors become more common, and the number of cores available on a processor increases, one can expect the speed of our function to grow almost proportionally to the number of cores, at least up to 16 cores. Finally, we point out that FFT networks are essentially "optimally parallelizable," and that our compression function has extremely small circuit depth, allowing it to be computed extremely fast in customized hardware.

\section{Properties of SWIFFT}

Here we review a number of statistical and cryptographic properties that are often desirable in hash functions, and discuss which properties our functions do and do not satisfy.

\subsection{Statistical Properties}

Here we review a number of many well-known and useful statistical properties that are often desirable in a family of hash functions, in both cryptographic and non-cryptographic applications (e.g., hash tables, randomness generation). All of these statistical properties can be proved unconditionally, i.e., they do not rely on any unproven assumptions about any computational problems.

Universal hashing. A family of functions is called universal if, for any fixed distinct $x, x^{\prime}$, the probability (over the random choice of $f$ from the family) that $f(x)=f\left(x^{\prime}\right)$ is the inverse of the size of the range. It is relatively straightforward to show that our family of compression functions is universal (this property is used implicitly in the proofs for the statistical properties below).

Regularity. A function $f$ is said to be regular if, for an input $x$ chosen uniformly at random from the domain, the output $f(x)$ is distributed uniformly over the range. More generally, the function is $\epsilon$-regular if its output distribution is within statistical distance (also known as variation distance) $\epsilon$ from uniform over the range. The only randomness is in the choice of the input.

As first proved in 18, our family of compression functions is regular in the following sense: all but an $\epsilon$ fraction of functions $f$ from the family are $\epsilon$-regular, 
for some negligibly small $\epsilon$. The precise concrete value of $\epsilon$ is determined by the particular parameters $(n, m, p)$ of the family.

Randomness extraction. Inputs to a hash function are often not chosen uniformly from the domain, but instead come from some non-uniform "real-world" distribution. This distribution is usually unknown, but may reasonably be assumed to have some amount of uncertainty, or min-entropy. For hash tables and related applications, it is usually desirable for the outputs of the hash function to be distributed uniformly (or as close to uniformly as possible), even when the inputs are not uniform. Hash functions that give such guarantees are known as randomness extractors, because they "distill" the non-uniform randomness of the input down to an (almost) uniformly-distributed output. Formally, randomness extraction is actually a property of a family of functions, from which one function is chosen at random (and obliviously to the input).

The proof of regularity for our functions can be generalized to show that they are also good randomness extractors, for input distributions having enough min-entropy.

\subsection{Cryptographic Properties}

Here we discuss some well-known properties that are often desirable in cryptographic applications of hash functions, e.g., digital signatures. Under relatively mild assumptions, our functions satisfy several (but not all) of these cryptographic properties. (For precise definitions, see, e.g., 23.)

Informally, a function $f$ is said to one-way if, given the value $y=f(x)$ for an $x$ chosen uniformly at random from the domain, it is infeasible for an adversary to find any $x^{\prime}$ in the domain such that $f\left(x^{\prime}\right)=y$. It is second preimage resistant if, given both $x$ and $y=f(x)$ (where $x$ is again random), it is infeasible to find a different $x^{\prime} \neq x$ such that $f\left(x^{\prime}\right)=y$. These notions also apply to families of functions, where $f$ is chosen at random from the family.

A family of functions is target collision resistant (also called universal oneway) if it is infeasible to find a second preimage of $x$ under $f$, where $x$ is first chosen by the adversary (instead of at random) and then the function $f$ is chosen at random from the family. Finally, the family is fully collision resistant if it is infeasible for an adversary, given a function $f$ chosen at random from the family, to find distinct $x, x^{\prime}$ such that $f(x)=f\left(x^{\prime}\right)$.

For functions that compress their inputs, the notions above are presented in increasing order of cryptographic strength. That is, collision resistance implies target collision resistance, which in turn implies second preimage resistance, which in turn implies one-wayness. All of the above notions are computational, in that they refer to the infeasibility (i.e., computational difficulty) of solving some cryptographic problem. However, the concrete effort required to violate these security properties (i.e., the meaning of "infeasible") will vary depending on the specific security notion under consideration, and is discussed in more detail below in Section 5 .

As shown in 2116, our family of compression functions is provably collision resistant (in an asymptotic sense), under a relatively mild assumption about 
the worst-case difficulty of finding short vectors in cyclic/ideal lattices. This in turn implies that the family is also one-way and second preimage resistant. In Section 5.1, we give a detailed discussion and interpretation of the security proofs. In Section 5.2, we discuss the best known attacks on the cryptographic properties of our concrete functions, and give estimates of their complexity.

\subsection{Properties Not Satisfied by SWIFFT}

For general-purpose cryptographic hash functions and in certain other applications, additional properties are often desirable. We discuss some of these properties below, but stress that our functions do not satisfy these properties, nor were they intended or designed to.

Pseudorandomness. Informally, a family of functions is pseudorandom if a randomly-chosen function from the family "acts like" a truly random function in its input-output behavior. More precisely, given (adaptive) oracle access to a function $f$, no adversary can efficiently distinguish between the case where (1) $f$ is chosen at random from the given family, and (2) every output of $f$ is uniformly random and independent of all other outputs. (The formal definition is due to [12.) We stress that the adversary's view of the function is limited to oracle access, and that the particular choice of the function from the family is kept secret.

Our family of functions is not pseudorandom (at least as currently defined), due to linearity. Specifically, for any function $f$ from our family and any two inputs $x_{1}, x_{2}$ such that $x_{1}+x_{2}$ is also a valid input, we have $f\left(x_{1}\right)+f\left(x_{2}\right)=$ $f\left(x_{1}+x_{2}\right)$. This relation is very unlikely to hold for a random function, so an adversary can easily distinguish our functions from random functions by querying the inputs $x_{1}, x_{2}$, and $x_{1}+x_{2}$. However, this homomorphism might actually be considered as a useful feature of the function in certain applications (much like homomorphic encryption).

With additional techniques, it may be possible to construct a family of pseudorandom functions (under suitable lattice assumptions) using similar design ideas.

Random oracle behavior. Intuitively, a function is said to behave like a random oracle if it "acts like" a truly random function. This notion differs from pseudorandomness in that the function is fixed and public, i.e., its entire description is known to the adversary. Though commonly used, the notion of "behaving like a random oracle" cannot be defined precisely in any meaningful or achievable way. Needless to say, we do not claim that our functions behave like a random oracle.

\section{$5 \quad$ Security Analysis}

In this section, we interpret our asymptotic proofs of security for collisionresistance and the other claimed cryptographic properties. We then consider cryptanalysis of the functions for our specific choice of parameters, and review the best known attacks to determine concrete levels of security. 


\subsection{Interpretation of Our Security Proofs}

As mentioned above, an asymptotic proof of one-wayness for SWIFFT was given in [18, and an asymptotic proof of collision-resistance (a stronger property) was given independently in 21 and 16. As in most cryptography, security proofs must rely on some precisely-stated (but as-yet unproven) assumption. Our assumption, stated informally, is that finding relatively short nonzero vectors in $n$-dimensional ideal lattices over the ring $\mathbb{Z}[\alpha] /\left(\alpha^{n}+1\right)$ is infeasible in the worst case, as $n$ increases. (See 182116] for precise statements of the assumption.)

Phrased another way, the proofs of security say the following. Suppose that our family of functions is not collision resistant; this means that there is an algorithm that, given a randomly-chosen function $f$ from our family, is able to find a collision in $f$ in some feasible amount of time $T$. The algorithm might only succeed on a small (but noticeable) fraction of $f$ from the family, and may only find a collision with some small (but noticeable) probability. Given such an algorithm, there is also an algorithm that can always find a short nonzero vector in any ideal lattice over the ring $\mathbb{Z}[\alpha] /\left(\alpha^{n}+1\right)$, in some feasible amount of time related to $T$ and the success probability of the collision-finder. We stress that the best known algorithms for finding short nonzero vectors in ideal lattices require exponential time in the dimension $n$, in the worst case.

The importance of worst-case assumptions in lattice-based cryptography cannot be overstated. Robust cryptography requires hardness on the average, i.e., almost every instance of the primitive must be hard for an adversary to break. However, many lattice problems are heuristically easy to solve on "many" or "most" instances, but still appear hard in the worst case on certain "rare" instances. Therefore, worst-case security provides a very strong and meaningful guarantee, whereas ad-hoc assumptions on the average-case difficulty of lattice problems may be unjustified. Indeed, we are able to find collisions in compression function of the related LASH- $x$ family of hash functions [3] by falsifying its underlying (ad-hoc) average-case lattice assumption (see Section 5.3).

At a minimum, our asymptotic proofs of security indicate that there are no unexpected "structural weaknesses" in the design of SWIFFT. Specifically, violating the claimed security properties (in an asymptotic sense) would necessarily require new algorithmic insights about finding short vectors in arbitrary ideal lattices (over the ring $\mathbb{Z}[\alpha] /\left(\alpha^{n}+1\right)$ ). In Section $[5.3$, we demonstrate the significance of our proofs by giving examples of two compression functions from the literature that look remarkably similar to ours, but which admit a variety of very easily-found collisions.

Connection to algebraic number theory. Ideal lattices are well-studied objects from a branch of mathematics called algebraic number theory, the study of number fields. Let $n$ be a power of 2 , and let $\zeta_{2 n} \in \mathbb{C}$ be a primitive $2 n$th root of unity over the complex numbers (i.e., a root of the polynomial $\alpha^{n}+1$ ). Then the ring $\mathbb{Z}[\alpha] /\left(\alpha^{n}+1\right)$ is isomorphic to $\mathbb{Z}\left[\zeta_{2 n}\right]$, which is the ring of integers of the so-called cyclotomic number field $\mathbb{Q}\left(\zeta_{2 n}\right)$. Ideals in this ring of integers (more generally, in the ring of integers of any number field) map to $n$-dimensional lattices under what is known as the canonical embedding of the number field. These 
are exactly the ideal lattices for which we assume finding short vectors is difficult in the worst case 2 Further connections between the complexity of lattice problems and algebraic number theory were given by Peikert and Rosen [22.

For the cryptographic security of our hash functions, it is important that the extra ring structure does not make it easier to find short vectors in ideal lattices. As far as we know, and despite being a known open question in algebraic number theory, there is no apparent way to exploit this algebraic structure. The best known algorithms for finding short vectors in ideal lattices are the same as those for general lattices, and have similar performance. It therefore seems reasonable to conjecture that finding short vectors in ideal lattices is infeasible (in the worst case) as the dimension $n$ increases.

\subsection{Known Attacks}

We caution that our asymptotic proofs do not necessarily rule out cryptanalysis of specific parameter choices, or ad-hoc analysis of one fixed function from the family. To quantify the exact security of our functions, it is still crucially important to cryptanalyze our specific parameter choices and particular instances of the function.

A central question in measuring the security of our functions is the meaning of "infeasible" in various attacks (e.g., collision-finding attacks). Even though our functions have an output length of about $n \lg p$ bits, we do not claim that they enjoy a full $2^{n \lg p}$ "level of security" for one-wayness, nor a $2^{(n \lg p) / 2}$ level of security for collision resistance. Instead, we will estimate concrete levels of security for our specific parameter settings. This is akin to security estimates for public-key primitives like RSA, where due to subexponential-time factoring algorithms, a 1024-bit modulus may offer only (say) a $2^{100}$ concrete level of security.

In Section 5.2. we describe the known algorithms to find collisions in our functions takes time at least $2^{106}$ and requires almost as much space. We also describe the known inversion attacks, which require about $2^{128}$ time and space.

Throughout this section, it will be most convenient to cryptanalyze our functions using their algebraic characterization as described in Section 2.1, and in particular, Equation (11).

Connection to Subset Sum. A very useful view of our compression function is as a subset sum function in which the weights come from the additive group $\mathbb{Z}_{p}^{n}$, and are related algebraically.

An element $\mathbf{a}$ in the ring $R=\mathbb{Z}_{p}[\alpha] /\left(\alpha^{n}+1\right)$ can be written as $a_{0}+a_{1} \alpha+$ $\ldots+a_{n-1} \alpha^{n-1}$, which we can represent as a vector $\left(a_{0}, \ldots, a_{n-1}\right) \in \mathbb{Z}_{p}^{n}$. Because $\alpha^{n} \equiv-1$ in the ring $R$, the product of two polynomials $\mathbf{a}, \mathbf{x} \in R$ is represented

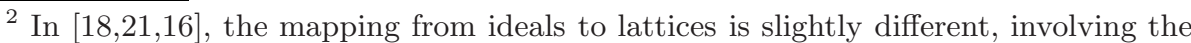
coefficient vectors of elements in $\mathbb{Z}\left[\zeta_{2 n}\right]$ rather than the canonical embedding. However, both mappings are essentially the same in terms of lengths of vectors, and the complexity of finding short vectors is the same under both mappings.
} 
by the matrix product of the square skew-circulant matrix of a with the vector representation of $\mathbf{x}$ :

$$
\mathbf{a} \cdot \mathbf{x} \in R \leftrightarrow\left[\begin{array}{cccc}
a_{0} & -a_{n-1} & \cdots & -a_{1} \\
a_{1} & a_{0} & \cdots & -a_{2} \\
\vdots & & \ddots & \\
a_{n-1} & a_{n-2} & \cdots & a_{0}
\end{array}\right]\left[\begin{array}{c}
x_{0} \\
x_{1} \\
\vdots \\
x_{n-1}
\end{array}\right] \bmod p
$$

Thus we can interpret Equation (1) (with fixed multipliers $\mathbf{a}_{1}, \ldots, \mathbf{a}_{m}$ ) as multiplying a fixed matrix $\mathbf{A} \in \mathbb{Z}_{p}^{n \times m n}$ by an input vector $\mathbf{x} \in\{0,1\}^{m n}$. The matrix A has the form

$$
\mathbf{A}=\left[\mathbf{A}_{1}|\cdots| \mathbf{A}_{m}\right]
$$

where each $\mathbf{A}_{i}$ is the $n \times n$ skew-circulant matrix of $\mathbf{a}_{i}$. Ignoring for a moment the algebraic dependencies within each $\mathbf{A}_{i}$, this formulation is equivalent to a subset sum function over the group $\mathbb{Z}_{p}^{n}$. Indeed, the output of our function is just the sum of a subset of the $m n$ column vectors of $\mathbf{A}$. And in fact, the fastest known algorithm for inverting (or finding collisions in) our function $f$ is the same one that is used for solving the high density subset sum problem [2815]. We describe this algorithm next.

Generalized Birthday Attack. Finding a collision in our function is equivalent to finding a nonzero $\mathbf{x} \in\{-1,0,1\}^{m n}$ such that

$$
\mathbf{A x}=\mathbf{0} \bmod p
$$

where $\mathbf{A}$ is as in Equation (4). This is because if we find a $\{-1,0,1\}$-combination of the columns of $\mathbf{A}$ that sums to $\mathbf{0} \bmod p$, the subset of the columns corresponding to the -1 s collides with the subset corresponding to the $1 \mathrm{~s}$. We will now describe an algorithm for finding such an $\mathbf{x}$ for the specific parameters $n=64$, $m=16, p=257$. Our goal is to provide a lower bound on the running time of the most efficient known algorithm for breaking our function. Therefore the analysis of the function will be fairly conservative.

Given a $64 \times 1024$ matrix $\mathbf{A}$ whose coefficients are in $\mathbb{Z}_{257}$, we proceed as follows:

1. Arbitrarily break up the 1024 column vectors of $\mathbf{A}$ into 16 groups of 64 vectors each.

2. From each group, create a list of $3^{64}$ vectors where each vector in the list is a different $\{-1,0,1\}$-combination of the vectors in the group.

We now have 16 lists each containing $3^{64} \approx 2^{102}$ vectors in $\mathbb{Z}_{257}^{64}$. Notice that if we are able to find one vector from each list such that their sum is the zero vector, then we can solve Equation 5 .

Finding one vector from each list such that the sum is $\mathbf{0}$ is essentially the $k$-list problem that was studied by Wagner [28], and is also related to the technique used by Blum, Kalai, and Wassserman [5] for solving the parity problem in the presence of noise. The idea is to use the lists to obtain new lists of vectors that 
are $\{-1,0,1\}$-combinations of $\mathbf{A}$ 's columns, but which have many coordinates that are 0 . We then continue forming lists in which the vectors have more and more coordinates equal to 0 . More precisely, we continue with the algorithm in the following way:

3. Pair up the 16 lists in an arbitrary way.

4. For each pair of lists $\left(L_{i}, L_{j}\right)$, create a new list $L_{i, j}$ such that every vector in $L_{i, j}$ is the sum of one vector from $L_{i}$ and one vector from $L_{j}$, and the first 13 positions of the vector are all 0 modulo 257.

There are a total of $257^{13} \approx 2^{104}$ different values that a vector in $\mathbb{Z}_{257}^{64}$ can take in its first 13 entries. Since the lists $L_{i}$ and $L_{j}$ each contain $3^{64} \approx 2^{102}$ vectors, there are a total of $2^{204}$ possible vectors that could be in $L_{i, j}$. If we heuristically assume that each of the $257^{13} \approx 2^{104}$ possible values of the first 13 coordinates are equally likely to occur 3 , then we expect the list $L_{i, j}$ to consist of $2^{204} \cdot 2^{-104}=2^{100}$ vectors whose first 13 coordinates are all 0 . For convenience, we will assume that the lists have $2^{102}$ vectors (this again is a conservative assumption that is in the algorithm's favor).

At the end of Step 4, we have 8 lists, each with $2^{102}$ vectors in $\mathbb{Z}_{257}^{64}$ whose first 13 coordinates are zero. We can now pair up these 8 lists and create 4 lists of $2^{102}$ vectors whose first 26 coordinates are zero. We continue until we end up with one list of $2^{102}$ elements whose first 52 coordinates are zero. This means that only the last 12 coordinates of these vectors may be nonzero. If the vectors are randomly distributed in the last 12 coordinates, then there should be a vector which consists of all zeros (because there are only $257^{12} \approx 2^{96}$ possibilities for the last 12 coordinates).

Since we started out with 16 lists of $2^{102}$ elements, the running time of the algorithm is at least $16 \cdot 2^{102}=2^{106}$. Notice that it also requires at least $2^{102}$ space.

Using a slightly modified generalized birthday attack, it is also possible to mount an inversion attack using time and space approximately $2^{128}$. The main difference is that we use 8 lists, and when combining (say) the first two lists in each level of the tree, we pair up entries so that they match the desired output value on the appropriate block of entries (for the other pairs of lists, we pair the entries to produce zeros).

Lattice Attacks. Lattice reduction is a possible alternative way to find a nonzero vector $\mathbf{x} \in\{-1,0,1\}^{m n}$ that will satisfy Equation (5). If we think of the matrix $\mathbf{A}$ as defining a linear homomorphism from $\mathbb{Z}^{m n}$ to $\mathbb{Z}_{p}^{n}$, then the kernel of $\mathbf{A}$ is $\operatorname{ker}(\mathbf{A})=\left\{\mathbf{y} \in \mathbb{Z}^{m n}: \mathbf{A y} \equiv \mathbf{0} \bmod p\right\}$. Notice that $\operatorname{ker}(\mathbf{A})$ is a lattice of dimension $m n$, and a vector $\mathbf{x} \in\{-1,0,1\}^{m n}$ such that $\mathbf{A x} \equiv \mathbf{0} \bmod p$ is a shortest nonzero vector in the $\ell_{\infty}$ or "max" norm of this lattice.

Because a basis for $\operatorname{ker}(\mathbf{A})$ can be computed efficiently given $\mathbf{A}$, finding a shortest nonzero vector (in the $\ell_{\infty}$ norm) of the lattice would yield a collision

${ }^{3}$ This is true if all the vectors in the lists are random and independent in $\mathbb{Z}_{257}^{64}$, but this is not quite the case. Nevertheless, since we are being conservative, we will assume that the algorithm will still work. 
in our compression function. The lattice $\operatorname{ker}(\mathbf{A})$ shares many properties with the commonly occurring knapsack-type lattice (see, e.g., [20]). Our lattice is essentially a knapsack-type lattice with some additional algebraic structure. It is worthwhile to note that none of the well-known lattice reduction algorithms take advantage of the algebraic structure that arises here. Because the dimension 1024 of our lattice is too large for the current state-of-the-art reduction algorithms, breaking our function via lattice reduction would require some very novel idea to exploit the additional algebraic structure. As things stand right now, we believe that the generalized birthday technique described in the previous section provides a more efficient algorithm for finding collisions in our function.

Viewing the kernel as a lattice also leads naturally to a relaxed notion of "pseudo-collisions" in our function, which are defined in terms other norms, e.g., the Euclidean $\ell_{2}$ norm or "Manhattan" $\ell_{1}$ norm. Note that for any actual collision corresponding to an $\mathbf{x} \in \operatorname{ker}(\mathbf{A})$, we have $\mathbf{x} \in\{-1,0,1\}^{m n}$ and therefore the $\ell_{2}$ norm of $\mathbf{x}$ is $\|\mathbf{x}\|_{2} \leq \sqrt{m n}$. However, not every nonzero $\mathbf{x} \in \operatorname{ker}(\mathbf{A})$ with $\|\mathbf{x}\|_{2} \leq \sqrt{m n}$ determines a collision in our function, because the entries of $\mathbf{x}$ may lie outside $\{-1,0,1\}$. We say that such an $\mathbf{x}$ is a pseudo-collision for the $\ell_{2}$ norm. More generally, a pseudo-collision for any $\ell_{p}$ norm $(1 \leq p<\infty)$ is defined to be a nonzero $\mathbf{x} \in \operatorname{ker}(\mathbf{A})$ such that $\|\mathbf{x}\|_{p} \leq(m n)^{1 / p}$. The set of pseudo-collisions only grows as $p$ decreases from $\infty$ to 1 , so finding pseudo-collisions using lattice reduction might be easier in norms such as $\ell_{2}$ or $\ell_{1}$. Finding pseudo-collisions could be a useful starting point for finding true collisions.

\subsection{Cryptanalysis of Similar Functions}

In this section, we briefly discuss two other compression functions appearing in the literature that bear a strong resemblance to ours, but which do not have asymptotic proofs of collision resistance. In fact, these compression functions are not collision resistant, and admit quite simple collision-finding algorithms. The attacks are made possible by a structural weakness that stems from the use of circulant matrices, which correspond algebraically to rings that are not integral domains. Interestingly, integral domains are the crucial ingredient in the asymptotic proofs of collision resistance for our function 2116. We believe that this distinction underscores the usefulness and importance of security proofs, especially worst-case hardness proofs for lattice-based schemes.

Micciancio's Cyclic One-Way Function. The provably one-way function described by Micciancio [18 is very similar to SWIFFT, and was the foundation for the subsequent collision-resistant functions on which this paper is based 2116]. Essentially, the main difference between Micciancio's function and the ones presented in 21, is is that the operations are performed over the ring $\mathbb{Z}_{p}[\alpha] /\left(\alpha^{n}-1\right)$ rather than $\mathbb{Z}_{p}[\alpha] /\left(\alpha^{n}+1\right)$. This difference, while seemingly minor, makes it almost trivial to find collisions, as shown in [21]6].

Just like ours, Micciancio's function has an interpretation as the product of a matrix $\mathbf{A}$ (as in Equation (4)) and a vector $\mathbf{x} \in\{0,1\}^{m n}$. The only difference 
is the matrices $\mathbf{A}_{i}$ from Equation (44) are circulant, rather than skew-circulant (i.e., just like Equation (3), but without negations).

Notice that in the vector product of a circulant matrix $\mathbf{A}_{i}$ with the all-1s vector $\mathbf{1}$, all of the entries are the same. Thus, for any circulant matrix $\mathbf{A}_{i}$, the $n$-dimensional vector $\mathbf{A}_{i} \cdot \mathbf{1} \bmod p$ can be only one of $p$ distinct vectors. There are $2^{m}$ ways to set each vector $\mathbf{x}_{1}, \ldots, \mathbf{x}_{m}$ to be either $\mathbf{0}$ or $\mathbf{1}$, but there are only $p$ distinct values of the compression function $\mathbf{A x}=\mathbf{A}_{1} \mathbf{x}_{1}+\ldots+\mathbf{A}_{m} \mathbf{x}_{m} \bmod p$. Because $2^{m}>p$ (otherwise the function does not compress), some pair of distinct binary vectors are mapped to the same output. Such a collision can be found in linear time.

LASH Compression Function. LASH- $x$ is a family of hash functions that was presented at the second NIST hash function workshop [3]. Its compression function $f_{\mathbf{H}}$ takes an $n$-bit input $\mathbf{x}=\mathbf{x}_{1} \mid \mathbf{x}_{2}$ where $\mathbf{x}_{1}, \mathbf{x}_{2} \in\{0,1\}^{n / 2}$, and is defined as $f_{\mathbf{H}}(\mathbf{x})=\left(\mathbf{x}_{1} \oplus \mathbf{x}_{2}\right)+\mathbf{H x} \bmod q$, where $\mathbf{H}$ is a "semi-circulant" $m \times n$ matrix whose entries are from the group $\mathbb{Z}_{256}$ :

$$
\mathbf{H}=\left[\begin{array}{ccccc}
a_{0} & a_{n-1} & a_{n-2} & \cdots & a_{1} \\
a_{1} & a_{0} & a_{n-1} & & a_{2} \\
\vdots & & & \ddots & \\
a_{m-1} & a_{m-2} & a_{m-3} & \cdots & a_{m}
\end{array}\right] .
$$

The values $a_{0}, \ldots, a_{n-1} \in \mathbb{Z}_{256}$ are essentially chosen at random (actually, according to a weak pseudorandom generator).

As discussed in [3], a heuristic assumption for the security of LASH is that its compression function $f_{\mathbf{H}}$ is collision-resistant. However, for a random choice of the entries $a_{0}, \ldots, a_{n-1}$, finding a collision in $f_{\mathbf{H}}$ with noticeable probability is actually trivial. Notice that all the rows of the matrix $\mathbf{H}$ have the same sum. If this sum happens to be $0 \bmod 256$ (which happens with probability $1 / 256$ over the choice of the $a_{i}$ ), then we have

$$
f(\mathbf{0})=0+\mathbf{H} \cdot \mathbf{0}=\mathbf{0}=0+\mathbf{H} \cdot \mathbf{1}=f(\mathbf{1}),
$$

where $\mathbf{0}$ and $\mathbf{1}$ are all-0s and all-1s vectors, respectively. Therefore these two distinct inputs make up a collision in the compression function.

When $n$ is divisible by a large power of 2 (e.g., $n=640,1024$ are proposed in [3]), other collisions may be easy to find as well (with some noticeable probability over the choice of the $a_{i}$ ). For example, the inputs $\mathbf{x}=0101 \cdots 01$ and $\mathbf{x}^{\prime}=1010 \cdots 10$ will collide with probability $1 / 256$, because $\mathbf{H} \mathbf{x}$ and $\mathbf{H x}^{\prime}$ consist of two repeated values, i.e., the sum of the even-indexed $a_{i}$ s and the sum of the odd-indexed $a_{i}$ s. Other kinds of collisions are also possible, corresponding essentially to the factorization of the polynomial $\alpha^{n}-1$ over $\mathbb{Z}[\alpha]$.

The attacks described above apply only to LASH's underlying compression function, and not (as far as we are aware) to the full LASH hash function itself. Using different ideas (that do not exploit the above-described structural weakness in $f_{\mathbf{H}}$ ), Contini et al [8] give a thorough cryptanalysis of the full LASH hash function. 


\section{Acknowledgments}

We thank Ron Steinfeld and Gaëtan Leurent (among others) for pointing out the more efficient inversion attacks using the generalized birthday attack.

\section{References}

1. Ajtai, M.: Generating hard instances of lattice problems. In: STOC, pp. 99-108 (1996)

2. Baritaud, T., Gilbert, H., Girault, M.: FFT hashing is not collision-free. In: Rueppel, R.A. (ed.) EUROCRYPT 1992. LNCS, vol. 658, pp. 35-44. Springer, Heidelberg (1993)

3. Bentahar, K., Page, D., Silverman, J., Saarinen, M., Smart, N.: Lash. Technical report, 2nd NIST Cryptographic Hash Function Workshop (2006)

4. Biham, E., Chen, R., Joux, A., Carribault, P., Jalby, W., Lemuet, C.: Collisions of SHA-0 and reduced SHA-1. In: Cramer, R.J.F. (ed.) EUROCRYPT 2005. LNCS, vol. 3494. Springer, Heidelberg (2005)

5. Blum, A., Kalai, A., Wasserman, H.: Noise-tolerant learning, the parity problem, and the statistical query model. Journal of the ACM 50(4), 506-519 (2003)

6. Cai, J., Nerurkar, A.: An improved worst-case to average-case connection for lattice problems. In: FOCS, pp. 468-477 (1997)

7. Camion, P., Patarin, J.: The knapsack hash function proposed at Crypto 1989 can be broken. In: Quisquater, J.-J., Vandewalle, J. (eds.) EUROCRYPT 1989. LNCS, vol. 434, pp. 39-53. Springer, Heidelberg (1990)

8. Contini, S., Matusiewicz, K., Pieprzyk, J., Steinfeld, R., Guo, J., Ling, S., Wang, H.: Cryptanalysis of LASH. Cryptology ePrint Archive, Report 2007/430 (2007), http://eprint.iacr.org/

9. Daemen, J., Bosselaers, A., Govaerts, R., Vandewalle, J.: Collisions for Schnorr's hash function FFT-hash presented at crypto 1991. In: Matsumoto, T., Imai, H., Rivest, R.L. (eds.) ASIACRYPT 1991. LNCS, vol. 739. Springer, Heidelberg (1993)

10. Damgård, I.: A design principle for hash functions. In: Brassard, G. (ed.) CRYPTO 1989. LNCS, vol. 435, pp. 416-427. Springer, Heidelberg (1990)

11. Goldreich, O., Goldwasser, S., Halevi, S.: Collision-free hashing from lattice problems. Technical Report TR-42, ECCC (1996)

12. Goldreich, O., Goldwasser, S., Micali, S.: How to construct random functions. J. ACM 33(4), 792-807 (1986)

13. Hoffstein, J., Pipher, J., Silverman, J.H.: NTRU: A ring-based public key cryptosystem. In: ANTS, pp. 267-288 (1998)

14. Joux, A., Granboulan, L.: A practical attack against knapsack based hash functions (extended abstract). In: De Santis, A. (ed.) EUROCRYPT 1994. LNCS, vol. 950, pp. 58-66. Springer, Heidelberg (1995)

15. Lyubashevsky, V.: The parity problem in the presence of noise, decoding random linear codes, and the subset sum problem. In: Chekuri, C., Jansen, K., Rolim, J.D.P., Trevisan, L. (eds.) APPROX 2005 and RANDOM 2005. LNCS, vol. 3624, pp. 378-389. Springer, Heidelberg (2005)

16. Lyubashevsky, V., Micciancio, D.: Generalized compact knapsacks are collision resistant. In: Bugliesi, M., Preneel, B., Sassone, V., Wegener, I. (eds.) ICALP 2006. LNCS, vol. 4052, pp. 144-155. Springer, Heidelberg (2006) 
17. Micciancio, D.: Almost perfect lattices, the covering radius problem, and applications to Ajtai's connection factor. SIAM J. on Computing 34(1), 118-169 (2004)

18. Micciancio, D.: Generalized compact knapsacks, cyclic lattices, and efficient oneway functions from worst-case complexity assumptions. Computational Complexity 16, 365-411 (2007); Preliminary version in FOCS 2002

19. Micciancio, D., Regev, O.: Worst-case to average-case reductions based on Gaussian measures. SIAM J. on Computing 37(1), 267-302 (2007)

20. Nguyen, P., Stehlé, D.: LLL on the average. In: ANTS, pp. 238-256 (2006)

21. Peikert, C., Rosen, A.: Efficient collision-resistant hashing from worst-case assumptions on cyclic lattices. In: Halevi, S., Rabin, T. (eds.) TCC 2006. LNCS, vol. 3876. Springer, Heidelberg (2006)

22. Peikert, C., Rosen, A.: Lattices that admit logarithmic worst-case to average-case connection factors. In: STOC, pp. 478-487; Full version in ECCC Report TR06-147 (2007)

23. Rogaway, P., Shrimpton, T.: Cryptographic hash-function basics: Definitions, implications, and separations for preimage resistance, second-preimage resistance, and collision resistance. In: Roy, B., Meier, W. (eds.) FSE 2004. LNCS, vol. 3017, pp. 371-388. Springer, Heidelberg (2004)

24. Schnorr, C.P.: FFT-hash, an efficient cryptographic hash function. In: Crypto Rump Session (1991)

25. Schnorr, C.P.: FFT-Hash II, efficient cryptographic hashing. In: Rueppel, R.A. (ed.) EUROCRYPT 1992. LNCS, vol. 658, pp. 45-54. Springer, Heidelberg (1993)

26. Schnorr, C.P.: Serge Vaudenay. Parallel FFT-hashing. In: Fast Software Encryption, pp. 149-156 (1993)

27. Vaudenay, S.: FFT-Hash-II is not yet collision-free. In: Brickell, E.F. (ed.) CRYPTO 1992. LNCS, vol. 740, pp. 587-593. Springer, Heidelberg (1993)

28. Wagner, D.: A generalized birthday problem. In: Yung, M. (ed.) CRYPTO 2002. LNCS, vol. 2442, pp. 288-303. Springer, Heidelberg (2002)

29. Wang, X., Lai, X., Feng, D., Chen, H., Yu, X.: Cryptanalysis for hash functions MD4 and RIPEMD. In: Cramer, R.J.F. (ed.) EUROCRYPT 2005. LNCS, vol. 3494. Springer, Heidelberg (2005)

30. Wang, X., Yu, H.: How to break MD5 and other hash functions. In: Cramer, R.J.F. (ed.) EUROCRYPT 2005. LNCS, vol. 3494. Springer, Heidelberg (2005) 